\title{
Tribute to Manuscript Reviewers
}

The number of manuscripts processed during the past 18 months has increased substantially and the content of many of those papers has become quite sophisticated. Each manuscript is reviewed by at least four referees. Hence, the identified journal reviewers on the masthead have been busy. In order to assist them as well as to ensure that papers are assessed by individuals knowledgeable in a similar field, to say nothing about the desire for a speedy turn-around time, the following individuals have been asked to bring their expertise to paper selection. The journal is most grateful to them for this volunteer activity.

I.R. Abbott, New York, N.Y. A.L. Albright, Pittsburgh, Pa. A.L. Amacher, Danville, Pa. P.A. Aronin, Detroit, Mich. W.O. Bell, Winston-Salem, N.C. S.E. Blaser, Toronto D.J. Bohn, Toronto DA. Bruce, Dallas, Tex. A.I. Canady, Detroit, Mich. D. Chitayat, Toronto D.D. Cochrane, Vancouver C.C. Duncan, New Haven, Conn. S.H. Ein, Toronto J. Goodrich, New York, N.Y. H J. Hoffman, Toronto H.E. James, San Diego, Calif. D.L. Johnson, Hershey, Pa. J.S. Kennerdell, Pittsburgh, Pa. 
J.P. Laurent, Houston, Tex.

T.B. Mapstone, Birmingham, Ala.

J.G. McComb, Los Angeles, Calif

J.P. Mickle, Gainesville, Fla.

G. Morrison, Miami, Fla.

S. Oi, Tokyo

A.D. Parent, Jackson, Miss.

T.S. Park, St Louis, Mo.

W.J. Peacock, Los Angeles, Calif.

J.H. Piatt, Jr, Portland, Oreg.

R.E. Pyeritz, Pittsburgh, Pa.

C. Raffel, Los Angeles, Calif.

D. Rotenstein, Pittsburgh, $\mathrm{Pa}$.

J.T. Rutka, Toronto

R.A. Sanford, Memphis, Tenn. P. Steinbok, Vancouver L.N. Sutton, Philadelphia, Pa. T. Tomita, Chicago, 111. 